\title{
Time Domain Reflectometry (TDR) technique - A solution to monitor moisture content in construction materials
}

\author{
Teresa Stingl Freitas ${ }^{1, *}$, Ana Sofia Guimarães ${ }^{1}$, Staf Roels $^{2}$, Vasco Peixoto de Freitas ${ }^{1}$, Andrea Cataldo $^{3}$ \\ ${ }^{1}$ Construct-LFC, Faculty of Engineering, University of Porto, R. Dr. Roberto Frias, 4200-465 Porto, Portugal \\ ${ }^{2}$ KU Leuven, Department of Civil Engineering, Building Physics Section, Kasteelpark Arenberg 40 Bus 2447,3001 Haverlee, Belgium \\ ${ }^{3}$ Department of Innovation Engineering, University of Salento, Via Monteroni, 73100 Lecce, Italy
}

\begin{abstract}
Measuring moisture content in building materials is crucial for the correct diagnosis of buildings' pathologies and for the efficiency evaluation of the treatment solution applied. There are several different techniques available to measure the moisture content in construction materials. However, perform long-term minor-destructive measurements is still a great challenge. The TDR - Time Domain Reflectometry technique is commonly used for moisture content measurements in soils, but is considered a relatively new method with regard to its application in construction materials. In the present state of research, the current use of the TDR technique for monitoring moisture content in all types of consolidated porous building materials is not possible yet. Indeed, the empirical conversion functions proposed for soils are mostly not suitable for building materials. Furthermore, to successfully use the TDR technique, a good contact between the TDR probe and the material under study is required, which may be difficult to achieve in hard materials. In this paper, the TDR technique was implemented in two limestone walls constructed in the lab to test experimentally the efficiency of a wall-base ventilation channel to speed up drying after a flood. Each wall was equipped with four two-rod TDR probes for continuous monitoring the moisture content in both situations: with and without the ventilation channel. All the equipment used, procedures followed during the drilling until the probes' final installation, as well as the individual calibration required for each probe are explained in detail. Instead of using unsuitable functions proposed for soils, the evaluation of the moisture content from the apparent relative dielectric permittivity measured was established using as reference method the gravimetric method. The results obtained suggest that the TDR technique is suitable for moisture content monitoring in consolidated porous building materials.
\end{abstract}

\section{Introduction}

The presence of high moisture content in building structures can lead to serious damage and needs to be avoided. Therefore, measuring moisture content in building materials is extremely important for the correct diagnosis of buildings' pathologies, for the adoption of appropriate intervention measures and for the efficiency evaluation of the treatment solutions applied. Despite the wide variety of techniques available for moisture content determination, performing continuous measurements of moisture content over time, in a minor destructive way, is still a great challenge. Indeed, most of the current methods require material sampling, and as a consequence successive measurements cannot be repeated in the same point, not to mention that the operation of sampling can change the real moisture content of the element under study. Another very common alternative, are the methods based on the measurement of surface moisture content. However, these non-destructive methods only give a partial information of the moisture content in the study element, taking into account that the water content near the surfaces are strongly influenced by the evaporation conditions $[1,2]$. As a matter of fact, many researchers end up using more pragmatic approaches to indirectly monitor the moisture content. One popular example is the use of relative humidity sensors, although they are not very suitable when it is intended to monitor high moisture content values.

Overall, perform accurate measurements of moisture content in building materials is a complex subject, which is still the focus of ongoing research. In this work, the TDR - Time Domain Reflectometry - technique is presented as a solution to continuous monitor the moisture content in construction materials in depth and in a minor destructive way. The application of the TDR technique in engineering and natural sciences has a relatively long history. It is a recognized technique in cable testing, widely used to measure dielectric properties of liquids mediums and it is also considered a well-established technique for moisture content measurements in soils. However, despite the advance and well-founded knowledge of the TDR technique as a measurement technology, its application for moisture content measurements in construction materials is considered a relatively new method [3].

\footnotetext{
*Corresponding author: tsf@fe.up.pt
} 


\section{Time Domain Reflectometry (TDR)}

\subsection{Basic principles}

The principle of any TDR measurement consists of launching an electromagnetic signal along the probe inserted into the material under test. The reflected signal carries information on the dielectric characteristics of the material in which the probe is inserted. Indeed, any impedance variation, found by the TDR signal when it propagates along the probe, causes the partial reflection of the propagating signal. The reflected signal, acquired by the TDR unit, is then analysed through a specific dataprocessing software, which provides a graphic called reflectogram. The reflectogram displays the reflection coefficient, $\rho$ (ratio between the amplitude of the reflected signal and the amplitude of the generated signal), as a function of the apparent distance, $\mathrm{d}_{\mathrm{app}}$. The quantity $\mathrm{d}_{\mathrm{app}}$ is not a real physical distance and its value is influenced by the travel time of the electromagnetic signal in the study medium. Figure 1 shows a TDR reflectogram obtained for a dry limestone with a $18,5 \mathrm{~cm}$ long two-rod probe. The first derivative of the TDR reflectogram is used to easily identify impedance variations and, in this way, represents a practical method for interface individuation. In the results obtained for the dry limestone, a first maximum peak corresponding to the connection between the coaxial cable and the probe-head can be clearly seen (Figure 1, point a)). Immediately after, a second minimum peak is detected corresponding to the interface between the probe-head end and the surface of the limestone under test (Figure 1, point b)). And finally, a last maximum peak is visible corresponding to the end of the probe (Figure 1, point c)). As a result, two electric lengths can be identified: $l_{h}$ - the apparent length introduced by the probe-head section - and $l_{r, M U T}$ - the apparent length of the probe that is actually in contact with the material under test (MUT): the rods.

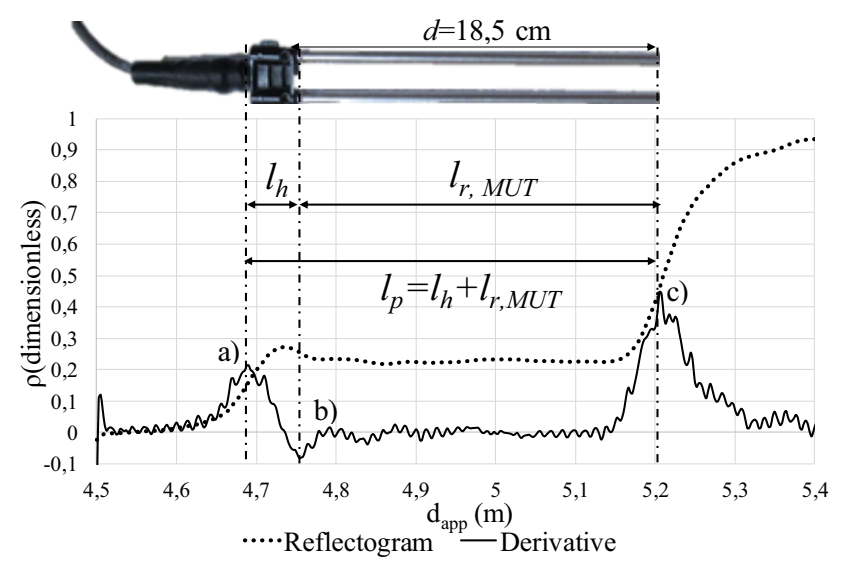

Fig. 1. TDR reflectogram and corresponding first derivative for a $18,5 \mathrm{~cm}$ long two-rod probe inserted in dry limestone. The peaks of the derivative, represented by the three vertical dashed lines, indicate the most significant impedance changes. Note: $l_{p}$ is the sum of the electric length of the probes' head and the electric length of the rods.
These results are used to obtain the apparent relative dielectric permittivity, $\varepsilon_{\text {app }}$, of the material under test at a certain instant of time, through the following equation [4]:

$$
\varepsilon_{\text {app }}=\left(l_{r, M U T} / d\right)^{2}
$$

Where $\mathrm{d}$ is the physical length of the probe used.

\subsection{Individual probes calibration}

For high-accuracy measurements the TDR probes require individual calibration, sometimes called as "probe-length calibration". The main reason is the fact that in Eq. (1) the physical length of the probe, $d$, measured with the tape maybe slightly different from the "probe electrical length" when it is placed in air, $l_{r, A I R}\left(\varepsilon_{\text {air }} \approx 1\right)$. This is mostly due to the presence of the probe head, taking into account that a small portion of the rods is contained inside the probehead. This detail needs to be properly compensated, otherwise it will lead to the presence of an "offset error". The apparent length of the rods in air, $l_{r, A I R}$, cannot be directly read from the reflectogram, because the interface between the probe-head end and the surface of the air (point b) in Figure 1) is very difficult to identify in the reflectogram obtained when the probe is placed in air (Figure 2).

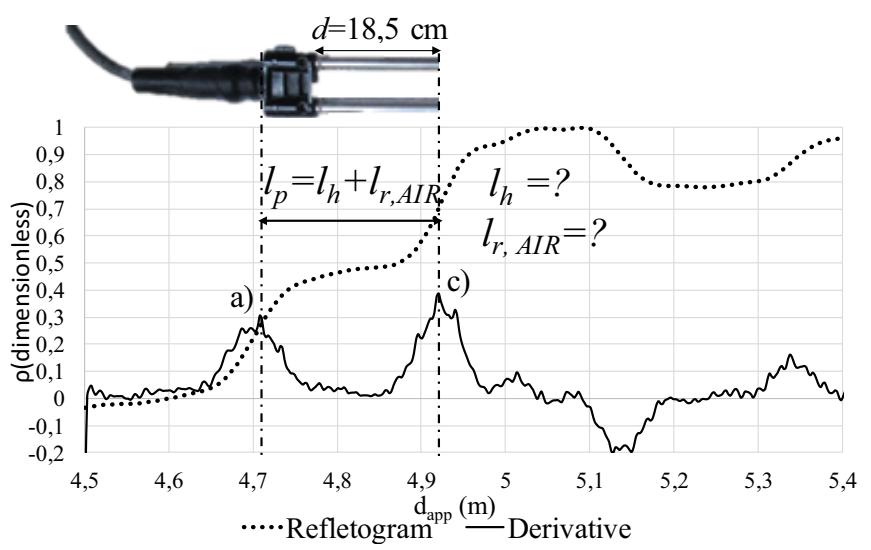

Fig. 2. TDR reflectogram and corresponding first derivative for a $18,5 \mathrm{~cm}$ long two-rod probe placed in air.

For this reason, the "probe-length calibration" consisted in carrying out ten repeated measurements with the probe inserted into materials with well-known dielectric permittivity. In this way, it is possible to determine the two unknown parameters for every probe: $l_{h}$ and $l_{r, A I R}$. This procedure needs to be repeated only once for each probe. Usually, the mediums chosen for this process are either water and air or water and benzene. Anyway, the permittivities of both materials should differ as much as possible to achieve the highest accuracy in the parameter determination. In our case, the measurements were done with the probes placed in air $\left(\varepsilon_{\mathrm{air}} \approx 1\right)$ and distilled water $\left(\varepsilon_{\text {water }} \approx 81\right.$, for a water temperature $\approx 17,5^{\circ} \mathrm{C}$ [5]). Using Eq. (1) and taking into account the equation $l_{p}=l_{h}+l_{r, M U T}$ (Figure 1), the two unknown parameters for each probe can be determined as follows: 


$$
\begin{cases}l_{p, \text { water }}=l_{h}+\sqrt{\varepsilon_{\text {water }}} \times l_{r, A I R} & \rightarrow l_{h}=\ldots \\ l_{p, \text { air }}=l_{h}+\sqrt{\varepsilon_{\text {air }}} \times l_{r, A I R} & \rightarrow l_{r, A I R}=\ldots\end{cases}
$$

Where $l_{p \text {,water }}$ and $l_{p \text {,air }}$ are known quantities read from the respective reflectograms (see Figure 2 as example).

Therefore, for high-accuracy measurements, the apparent relative dielectric permittivity, $\varepsilon_{\mathrm{app}}$, of the material under test should be determined according to the following equation:

$$
\varepsilon_{\text {app }}=\left(l_{r, M U T} / l_{r, A I R}\right)^{2}
$$

instead of using equation (1).

\subsection{Suitability to detect moisture content variations}

The apparent relative dielectric permittivity measurements, $\varepsilon_{\text {app }}$, can be used to infer the moisture content in building materials, because the travel time of the electromagnetic signal is influenced by the presence of water. Indeed, as the relative dielectric permittivity of water $\left(\varepsilon_{\text {water }} \approx 78-82\right)$ is considerably higher than the relative permittivity of many dry building materials ( $\varepsilon_{\text {dry }}$ builiding materials $\approx 2-9$ ), the presence of water leads to an increase of the overall dielectric permittivity of the material under test [6,7]. Figure 3 shows the reflectograms obtained in three extreme scenarios. As expected, the measurements support and are in agreement with the hypothesis mentioned above. The total apparent length of the probe obtained in air, $l_{p, A I R}$, is smaller than the one

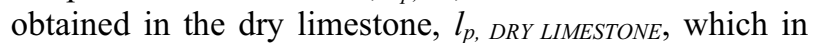
turn is smaller than the obtained in water, $l_{p, W A T E R}$.

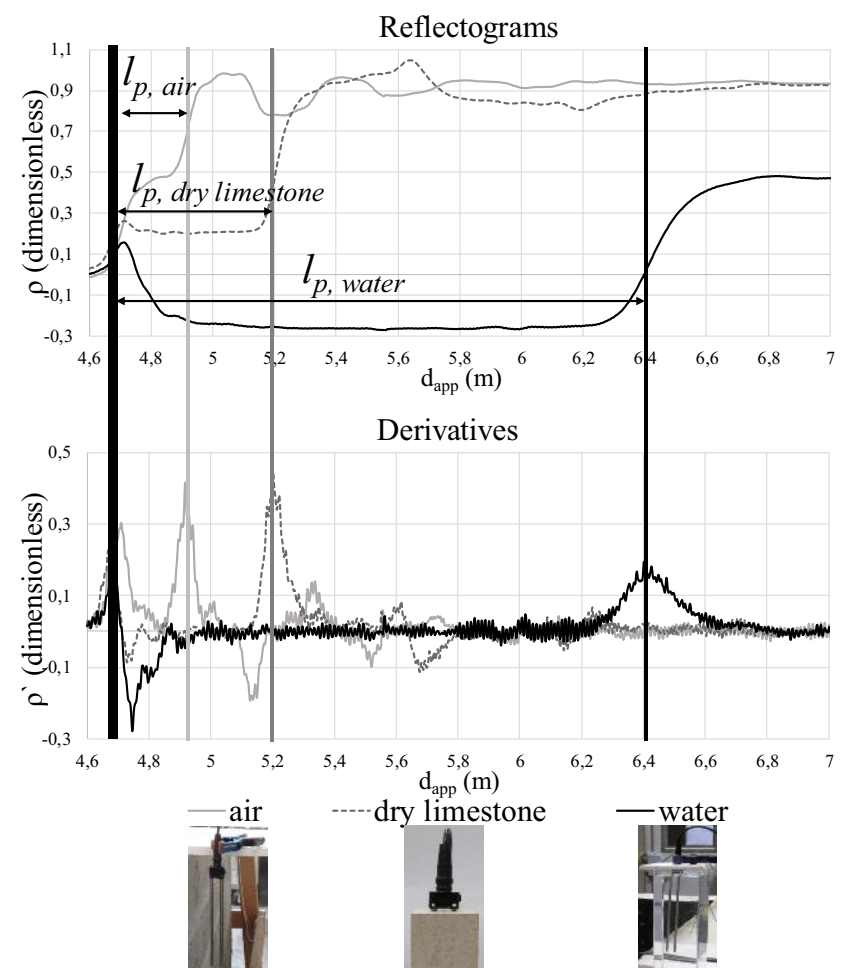

Fig. 3. TDR reflectograms and corresponding first derivatives obtained for air, dry limestone and water.
Therefore, in TDR measurements performed in building materials with increasing moisture content, the apparent length of the probes is expected to be progressively longer and consequently higher values of $\varepsilon_{\text {app }}$ should be obtained. The difference between the $\varepsilon_{\text {app }}$ value collected when the material is completely dry or saturated will depend on the material's porosity. In high porous building materials this range will be bigger.

\subsection{Approaches to determine the moisture content from the measured apparent relative permittivity}

There are three basic approaches to calculate the moisture content from the measured apparent relative permittivity:

- Empirical conversion functions developed for soil moisture measurements. The most common and popular conversion function is the third order polynomial relation proposed by Topp et al. in 1980, which is expressed by the following equation:

$$
\begin{aligned}
& w=-5,3 \times 10^{-2}+2,92 \times 10^{-2} \varepsilon_{\mathrm{app}} \\
& -5,5 \times 10^{-4} \varepsilon_{\mathrm{app}}^{2}+4,3 \times 10^{-6} \varepsilon_{\mathrm{app}}^{3}
\end{aligned}
$$

Where $w$ is the moisture content in the porous body $\left(\mathrm{m}^{3} / \mathrm{m}^{3}\right)$. A few years later, in 1996, Malicki et al. proposed a more generalized function, which takes into account the changes in the bulk density, $\rho\left(\mathrm{kg} / \mathrm{m}^{3}\right)$, of the dry material:

$$
w=\frac{\sqrt{\varepsilon_{\mathrm{app}}}-0,819+0,168 \rho-0,159 \rho^{2}}{7,18+1,18 \rho}
$$

The utilization of empirical conversion functions is very easy and therefore many researchers have already test their applicability to building materials. Unfortunately, the obtained results with both these relations failed completely in the moisture content determination of several materials: gypsum [8], calcium silicate [7], autoclaved aerated concrete and sandstone [9]. As a result, the exiting calibration functions for soils are mostly considered as not suitable for construction materials.

- Empirical conversion functions based on dielectric mixing models. The use of dielectric mixing models involves knowing the relative permittivities of the particular constituents that compose the materials, which are typically: the solid phase, the liquid phase (water) and the gaseous phase (air), as well as some other parameters that cannot be measured directly but have to determine by empirical calibration of the model. There are a variety of mixing formulas available to describe the relative permittivity of the whole material from its constituents. In Eq. (7) the 4-phase $\alpha$-model proposed by Dobson et al. is presented as an example.

$$
w=\frac{\varepsilon_{e f f}^{\alpha}-w_{b w}\left(\varepsilon_{b w}^{\alpha}-\varepsilon_{f w}^{\alpha}\right)-(1-\psi) \varepsilon_{s}^{\alpha}-\psi \varepsilon_{a}^{\alpha}}{\varepsilon_{f w}^{\alpha}-\varepsilon_{a}^{\alpha}}
$$


Where $\varepsilon_{\text {eff }}$ is the measured value of the relative permittivity of the porous medium, $w_{\mathrm{bw}}$ the amount of water bound on pore walls $\left(\mathrm{m}^{3} / \mathrm{m}^{3}\right), \varepsilon_{\mathrm{bw}}$ the relative permittivity of the bound water $\left(\varepsilon_{\mathrm{bw}} \approx 3,1\right), \varepsilon_{\mathrm{fw}}$ the relative permittivity of free water $\left(\varepsilon_{\mathrm{fw}} \approx 78-82\right.$, depending on the water temperature), $\varepsilon_{\mathrm{a}}$ the relative permittivity of air $\left(\varepsilon_{\mathrm{a}} \approx 1\right), \psi$ the total open porosity and $\alpha$ is an empirical parameter. In this model the significant difference between the relative permittivity of free and bound water is taken into account, which is an important step for a more accurate determination of moisture content in building materials using TDR measurements. Indeed, unlike most soils, building materials usually contain a significant amount of hygroscopic moisture and its inclusions into the conversion formulas represents a better approximation than the empirical conversion functions proposed for soils. Despite the promising advantages of the dielectric mixing models, further tests in a wide range of different building materials are still required to find generalized functions for distinct class of materials [10].

- Empirical calibration for the particular material under study using a reference method, such as the gravimetric method. In this case, the relation between the moisture content and the measured apparent relative permittivity is established for the precise material under test. This method is the most reliable until now but also the most time consuming one [3].

In this study, the calibration will be done according to this last method. A more detailed description of the procedure followed, as well as some of the results obtained are presented in sections 3.3. and 4. In the near future, and after collecting more results, the goal is to present a new empirical calibration curve for limestone $\left(\mathrm{w}\left(\varepsilon_{\mathrm{app}}\right)\right)$, which maybe then used for further testing and validation of several calibration function based on dielectric mixing models

\subsection{TDR advantages and disadvantages for moisture monitoring in buildings materials}

Monitoring moisture content in building materials is a complex subject, which is still the focus of ongoing research. Unlike the most common moisture methods, which require material sampling and are only capable to provide spot measurements at a certain instant of time, the TDR technique enables continuous long-term minor destructive monitoring of moisture content in construction materials. Furthermore, many common moisture measurement techniques can only be employed in the laboratory and are not suitable to be use on site due to the requirements of the equipment involved, as happens with the nuclear magnetic resonance method (NMR) or with the radiation attenuation techniques, like $\mathrm{x}$-ray, neutron absorption and $\gamma$-ray attenuation method [11]. As a matter of fact, and according to the research work done, the TDR technique has also never been used for walls in situ. The main obstacle in this case would not be the equipment but the difficulty of establishing a calibration curve $\left(\varepsilon_{\text {app }}(\mathrm{w})\right)$ when the material is not homogeneous. However, as the TDR technique is capable of monitoring dynamic changes fast and with accuracy (i.e. recording changes rather than static measurements) the need for calibration can be considered less important for some practical applications. For many professionals, the ability to monitor relative changes in the moisture content over time is more than enough to evaluate the efficiency of several design solutions made by civil engineers.

As for the disadvantages of the TDR technique, three relevant features deserve to be highlighted. The first one is the need to carry out an individual "probe-length calibration" before made any moisture measurements to ensure high-accuracy results. This procedure can be done in advance all at once for every single probe and is independent of the material under study. The second one is the difficulty of getting a suitable relation between the moisture content and the measured relative permittivity for the construction material under test. The empirical conversion functions proposed for soils are mostly not appropriate for building materials and the dielectric mixing formulas are not yet available for general classes of materials. Therefore, the most reliable method for now is still the use of conversion functions established for the particular material under test using a reference method. However, this is a very time consuming task, which constitutes an obstacle to the frequent use of the TDR technique in construction materials. The third and last difficulty is the need to ensure a good contact between the TDR probe rods and the material under test. Indeed, any air gap present between the waveguides and the material will compromise the moisture measurements. Drilling regular parallel holes with accuracy, without producing a large air gap, is very difficult particularly for hard materials. That is why most of the research work about the use of the TDR technique for moisture measurement was done for loose, granular, soft construction materials [7, 12-13]. As a matter of fact, many researchers recommend the use of the powder, gathering during the drilling, to fix the probes into the holes and in this way prevent the appearance of the unwanted air layer [12].

\section{TDR application}

The TDR technique was implemented in two limestone walls constructed in the laboratory. The final goal is to use the continuous moisture content measurements provided by the TDR technique to experimentally test the efficiency of a wall-base ventilation channel to speed up drying after a flood. The wall-base ventilation system has already been successfully studied in previous works, where it proved to be efficient to reduce rising damp problems in historic buildings [14-15]. The climate change witnessed during the last decades have generated new and unpredictable threats to this type of buildings, such as floods. Indeed, floods can lead to persistent consequences for the buildings' performance and irreversible damages to historic heritage, which can be minimized through the development of protective measurements. For that reason, study the effectiveness of 
the system to accelerate the drying process after a flood seems to be of great importance. Figure 4 shows a picture with the experimental set-up developed.

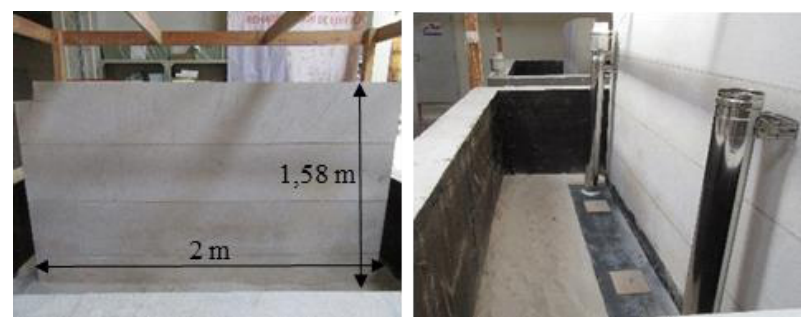

Fig. 4. Two limestone walls built in the laboratory: one with and one without the wall-base ventilation system. The limestone is a material typically used in the construction of historic buildings in Portugal.

Each wall is composed of five layers of limestone, each $20 \mathrm{~cm}$ high, linked by $1 \mathrm{~cm}$ thick lime mortar joints. Both walls have the same dimensions $(2 \mathrm{~m} \times 1,58 \mathrm{~m} \times$ $0,20 \mathrm{~m}$ ) and were built inside large tanks, in order to simulate a flood. The fans connected to the ventilation channel (to test forced drying) were placed in a higher position through the use of metallic conducts (see picture at the right in Figure 4). The drying phenomenon in the limestone walls will be studied both with and without the ventilation channel to test its efficiency.

\subsection{Equipment}

The TDR measurements were performed through a portable reflectometer from Campbell Scientific: the TDR200 [16]. The TDR200 unit generates a short time electromagnetic pulse that is applied to the probes inserted into the material under test. One of the advantages of this TDR instrument is that it supports multiplexers, which allows the simultaneous connection of several probes to a single TDR unit. In this work, one eight-channel multiplexer also from Campbell Scientific was used (SDM8X50), which will allow the continuous monitoring of moisture content in eight points in the walls. The probes were especially designed for this research (taking into account the wall thickness) and were fabricated by a spinoff company of the University of Salento - MoniTech. Each probe is made of two $18,5 \mathrm{~cm}$ long parallel stainless rods separated by $19 \mathrm{~mm}$ with a diameter of $6 \mathrm{~mm}$ (Figure 5). To analyse the reflected signal an appropriate Labview-based software was used. The direct output of the software is a reflectogram, as was detailed explained in section 2.1.

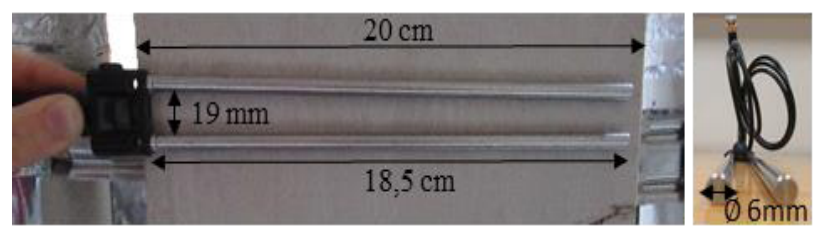

Fig. 5. Picture of the two-rod probe used for the TDR measurements (especially designed for the wall thickness).

\subsection{Installation steps}

One of the major obstacles pointed out by several authors to the use of the TDR technique in hard materials is the difficulty of ensuring a good contact between the TDR rods and the material under test (see section 2.4). To overcome this difficulty, a steel auxiliary guide was developed (Figure 6). This device was designed for the particular probes used in this work and is composed of two independent pieces.

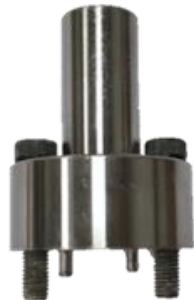

(a)

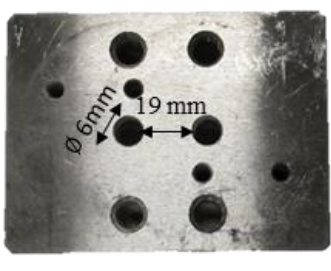

(b)

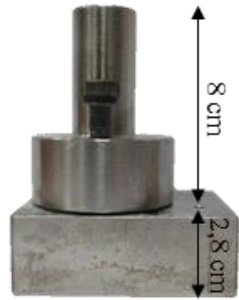

(c)
Fig. 6 Steel auxiliary guide composed of two separate pieces: (a) Side view of the first piece (b) top view of the second piece (c) entire guide.

To insert the TDR probes into the walls the steel guide was fixed to the surface of the wall during the drilling to ensure parallel and appropriate spaces between the holes. Figure 7 shows all steps followed during the drilling until the probes final installation.

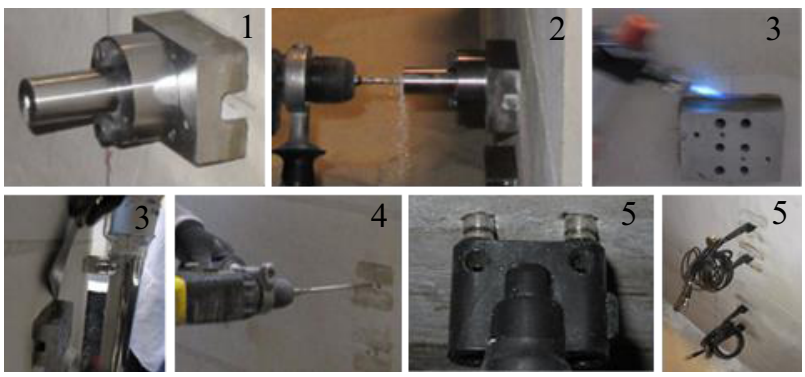

Fig. 7 Steps followed during the drilling until the probes final installation: 1) the guide was fixed to the surface of the wall with a resistant glue; 2) the drilling was done until the power drill hit the guide; 3 ) the guide was removed by heating the glue; 4) the drilling continued until reach $18,5 \mathrm{~cm}$ depth; 5) the probes were completely inserted into the walls.

This procedure is time-consuming, particularly for hard material like limestone, and needs to done with care because the lack of consistency between the holes bored to accommodate the rods can lead to variations in the moisture content measurements. Figure 8 shows the final experimental set-up.
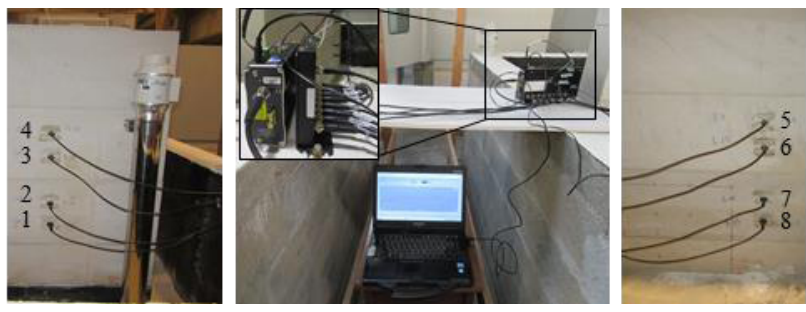

Fig. 8. Final experimental set-up for monitoring moisture content using the TDR method (left wall with the ventilation channel and right wall without). 
Each wall was equipped with four two-rod TDR probes placed at different heights for continuous monitoring the moisture content both with and without the ventilation channel. The preliminary results obtained on the dry walls for each of the eight probes installed, were already presented in a previous work [17]. They revealed that no air layer was present between the rods and the limestone and, therefore, the installation was considered a success.

\subsection{Calibration}

The relation between the apparent relative dielectric permittivity, $\varepsilon_{\text {app }}$, and the moisture content, w, for the limestone was established using the gravimetric method as reference, taking into account the reasons explained in section 2.3. For this purpose, a block was cut from the wall and several small prism-shaped samples of limestone $(20 \mathrm{~cm} \times 6 \mathrm{~cm} \times 6 \mathrm{~cm})$ were prepared (Figure 9). The same drilling procedure, previously described for the walls, was also followed for these samples.
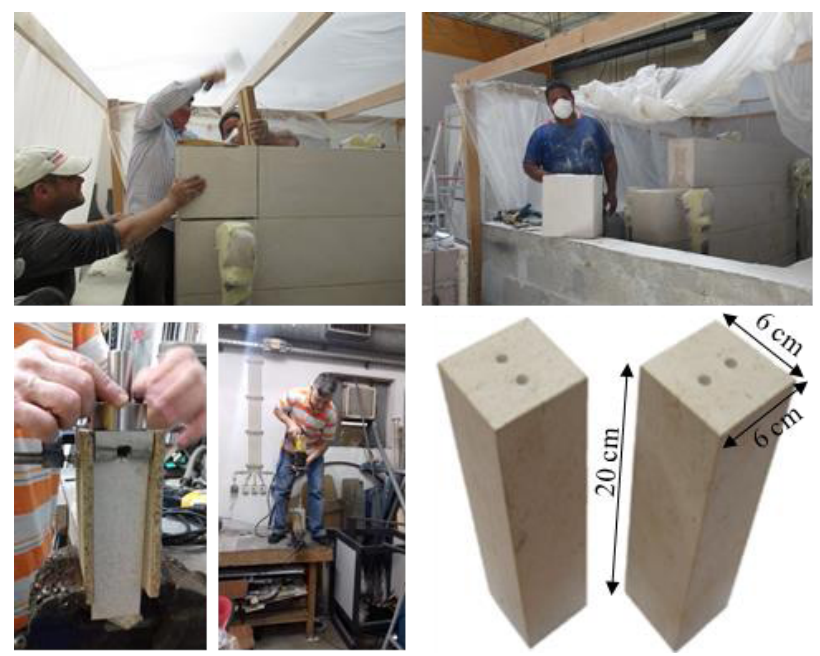

Fig. 9. Limestone samples for gravimetrical calibration.

Figure 10 shows a picture for each of the six steps defined for the calibration procedure.

First, the samples have been immersed in water for a certain predefined period of time. After this period, the samples were taken out of the water and their surfaces dried with a damp sponge, to remove the excess of water, before weighing. Immediately after, the samples were water and water vapour insulated at all sides, to ensure that no water would evaporate from the sample. In the measurements already made, the samples were first isolated with a plastic film (typically used in the kitchens) and then with aluminium tape. The plastic film was used to ensure that the aluminium tape did not damage the samples and so the process could be repeated for other moisture content values. After waiting for several days, to ensure redistribution of the moisture content in the samples, they were weighed again to guarantee that no water has evaporated and a TDR measurement can be finally made. The TDR measurements were repeated until the measured values of the apparent relative dielectric permittivity reached a constant value.
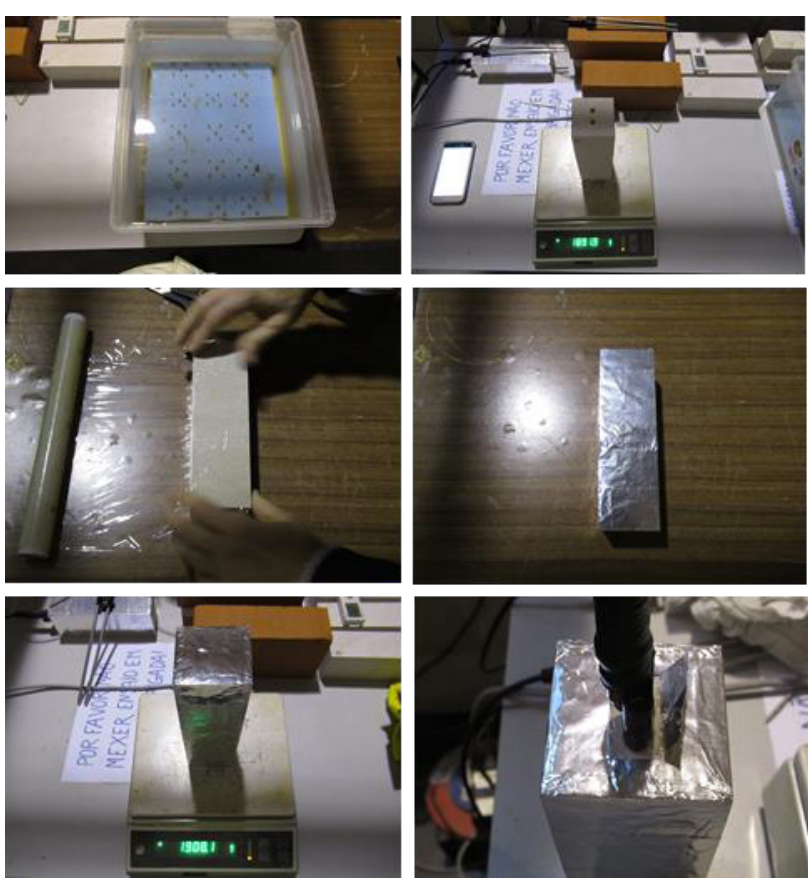

Fig. 10. Six steps followed for the gravimetrical calibration.

All these steps should be repeated for different immersion times in water or, in other words, for different moisture content values to obtain a calibration curve $\mathrm{w}\left(\varepsilon_{\mathrm{app}}\right)-$ for limestone.

\section{Results and discussion}

Figure 11 shows the reflectograms acquired for one limestone sample in three different scenarios: when the sample was completely saturated, after 100 minutes of immersion in water and after removing the sample form the oven. The second reflectogram was obtained according to the steps described in section 3.3. The results revealed that the TDR technique is suitable to detect moisture content variations in the limestone. Despite their low porosity, whenever the moisture content increases in the study material, the greater is the apparent distance corresponding the probe's length.

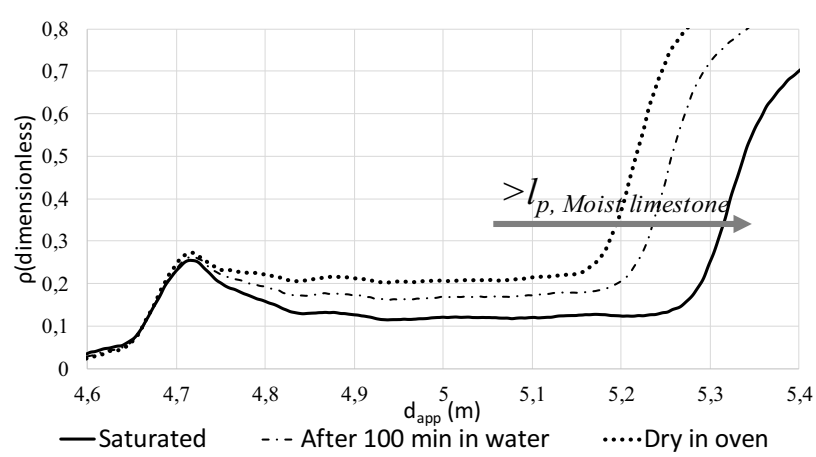

Fig. 11. TDR reflectograms acquired for the same limestone sample for three different scenarios: saturated, partially moist and dry.

Another important observation is related to the reflection coefficient value, $\rho$. It shows an almost constant value in the portion of the reflectograms corresponding to the probe's length. This result is particularly important for 
the measurement obtained with the dry sample, because it revealed that no air layer was present between the rods and the limestone, and for the measurement recorded after $100 \mathrm{~min}$ in water, because it revealed that the moisture content was already redistributed throughout the sample. As the behaviour of $\rho$ is strictly associated with the impedance variations encountered by the propagating electromagnetic signal along the probe, a constant value of $\rho$ means that the dielectric characteristics of the limestone are practically uniform.

For each value of moisture content study $\left(w_{\text {asturated }}=30,91 \mathrm{~kg} / \mathrm{m}^{3} ; w_{\text {after }} 100 \mathrm{~min}\right.$ in water $=14,59 \mathrm{~kg} / \mathrm{m}^{3}$ and $\mathrm{W}_{\text {oven }}=0 \mathrm{~kg} / \mathrm{m}^{3}$ ), ten repeated TDR measurements were performed. The values of $l_{r \text {,LIMESTONE }}$ were averaged and the corresponding apparent relative dielectric permittivity, $\varepsilon_{\text {app }}$, was evaluated by applying:

$$
\varepsilon_{\text {app }}=\left(\frac{\frac{1}{10} \sum_{i}^{10} l_{r, \text { LIMESTONE }}}{l_{r, A I R}}\right)^{2}
$$

Table 1 shows the relation between the moisture content and the apparent relative dielectric permittivity obtained for the same limestone sample.

Table 1. Relation between the moisture content and the apparent relative dielectric permittivity for limestone.

\begin{tabular}{|c|c|c|c|}
\hline & Sample weight $(\mathrm{g})$ & $\mathrm{w}\left(\mathrm{kg} / \mathrm{m}^{3}\right)$ & $\varepsilon_{\text {app }}(-)$ \\
\hline Dry in oven & 1891,9 & 0 & 6,8 \\
\hline Moist & 1902,3 & 14,59 & 7,3 \\
\hline Saturated & 1914,6 & 30,91 & 8,3 \\
\hline
\end{tabular}

The difference between the $\varepsilon_{\text {app }}$ value for the dry and saturated limestone is small. This is mainly due to the low porosity of the material. In spite of that, the technique proved to be suitable to detect even smaller moisture content variations $\left(\varepsilon_{\mathrm{app}}=7,3\right.$ for $\left.\mathrm{w}=14,59 \mathrm{~kg} / \mathrm{m}^{3}\right)$.

In the near future, the relation between $\mathrm{w}$ and $\varepsilon_{\text {app }}$ will be established for more points, in order to adjust a polynomial function to the measured data and in this way propose a calibration function for limestone. In addition, these tests will be performed in more samples to evaluate the reproducibility of the results.

\section{Conclusions}

Monitoring moisture content in building materials is a complex subject, which is still the focus of ongoing research. Despite the wide use of the TDR technique, especially for moisture content measurements in soils, the application in construction materials is considered a relatively new method. One of the major problems pointed out by several authors to the use of the TDR technique in hard construction materials is the difficulty of ensuring a good contact between the TDR probe and the medium. In addition, the empirical conversion functions used in soil moisture measurements are mostly not suitable for building materials and in the current state of research general formulas for determination of moisture content from the measured apparent relative permittivity are not yet available, especially for low porous building materials. Therefore, the establishment of a calibration curve for the particular material under test, using a reference method, is so far the most reliable solution. However, this is a time consuming procedure and represents another obstacle to use the TDR technique in construction materials.

This work aims to contribute to solve these two problems by testing the suitability of the TDR technique for moisture content measurements in limestone. The results obtained so far revealed that:

- The steel auxiliary guide developed allow drilling parallel and appropriate holes to accommodate the probes. As a result, no air gap was present next to the rods and the installation was considered a success;

- The technique is sensible to detect moisture content variations in limestone (despite their low porosity) and even small moisture content variations (Figure 11).

The intention, in the near future, is to present a new empirical calibration function valid for limestone that can also be used for further tests and validation of more general functions for similar types of low porous building materials (more measurements in Table 1).

The final main goal, after getting the calibration curve, will be test the suitability of the TDR technique for continuous long-term monitoring of moisture content in real-scale limestone walls (Figure 4).

This work was financially supported by: Base Funding UIDB/04708/2020 of the CONSTRUCT - Instituto de I\&D em Estruturas e Construções - funded by national funds through the FCT/MCTES (PIDDAC). Teresa Stingl Freitas would like to thank FCT for financial support through the grant SFRH/BD/121549/2016.

\section{References}

1. M. Phillipson, P. Baker, M. Davies, Z.Ye, A. McNaughtan, G.Galbraith, R. Mclean, Building Serv. Eng. Res. Technol., 28, 303-316 (2007)

2. M. Said, International Journal of Architectural Heritage , 1, 293-310 (2007)

3. R. Cerny, Measurement, 42, 329-336 (2009)

4. A. Cataldo, E. Benedetto, G. Cannazza, Synthesis Lectures on Emerging Engineering Technologies, 2,1-96 (2016)

5. U. Kaatze, Measurement Science and Technology, 18, 967-976 (2017)

6. Z. Suchorab, H. Sobczuk, R. Cerny, Z. Pavlik, R.S. Miguel, Environ. Protect. Eng., 35, 179-189 (2009).

7. Z. Pavlik, J. Mihulka, L. Fiala, R. Cerny, Int. J. Thermophys, 33, 1661-1673 (2012)

8. Z. Pavlik, L. Fiala, R. Cerny, J. Appl. Sci, 8, 17321737 (2008)

9. M. Phillipson, P. Baker, M. Davies, Z.Ye, G. Galbraith, R. Mclean, Building Serv. Eng. Res. Technol., 29, 261-272 (2008)

10. Z. Pavlik, L. Fiala, R. Cerny, Pollack Periodica, 4, 79-88 (2009) 
11. S. Pinchin, Studies in Conservation, 53, 33-45 (2008)

12. Z. Pavlik, M. Jirickova, R. Cerny, H. Sobczuk, Z. Suchorab, Journal of Building Physics, 30, 59-70 (2006)

13. A. Cataldo, E. Benedetto, G. Cannazza, E. Piuzzi, IEEE Transactions on Instrumentation and Measurement, 67, 1230-1237 (2017)

14. I. Torres, V. P. Freitas, Building and Environment, 42, 424-435 (2007)

15. A. S. Guimarães, J. Delgado, V. P. Freitas, Heat and Mass transfer, 48, 2079-2085 (2012)

16. TDR200 Instructional Manual - revision 4/17 (2017). https://s.campbellsci.com/documents/ca/manuals/tdr 200 man.pdf [online available].

17. T.S. Freitas, A.S. Guimarães, V.P. Freitas, MATEC web of conferences, 282, 02051 (2019) 